


\section{Les professeurs de langues étrangères, ambassadeurs du plurilinguisme et des échanges internationaux : résultats de l’Enquête TALIS 2018}

- En moyenne, dans les pays et économies participant à TALIS, $18 \%$ des enseignants enseignent les langues vivantes étrangères. La plupart des professeurs de langues vivantes étrangères (63 \%) enseignent aussi en parallèle une autre catégorie de matières.

- En moyenne, dans les pays et économies participant à TALIS, les professeurs de langues étrangères sont plus susceptibles que les autres enseignants de s'estimer préparés à l'enseignement en milieu multiculturel ou plurilingue, et d'indiquer que cet aspect figurait dans leurs études ou leur formation dans le cadre institutionnel.

- Les professeurs de langues étrangères ont un éventail plus large d'expériences de mobilité internationale que les autres enseignants, notamment en termes de voyages avec leurs élèves : $24 \%$ des professeurs de langues étrangères ont accompagné des élèves en voyage à l'étranger (contre $13 \%$ des autres enseignants), en moyenne, dans les pays et économies participant à TALIS qui ont administré cette question.

- Les professeurs de langues étrangères sont plus susceptibles (de 6 points de pourcentage) que les autres enseignants d'utiliser les TIC en classe, en moyenne, dans les pays et économies participant à TALIS. Ils sont de ce fait peut-être mieux préparés que les autres enseignants à utiliser les TIC pour permettre aux élèves d'accéder à des contenus multiculturels et plurilingues en période de restriction des déplacements.

Dans le monde d'aujourd'hui, la mondialisation, l'innovation technologique et les migrations humaines ont rendu presque inévitables les interactions entre personnes de différents pays et cultures. Dans ce contexte, la capacité à communiquer dans plus d'une langue est devenu une compétence clé présentant d'importants avantages économiques pour les individus et les économies. Cependant, l'intérêt de l'apprentissage d'autres langues va au-delà de la simple amélioration de la communication : il favorise également la compréhension de la complexité des cultures et des langues, et permet aux élèves de découvrir d'autres visions du monde. Ce sont là des préalables importants à une participation active à notre monde de plus en plus globalisé. L'apprentissage d'une langue étrangère peut donc constituer un puissant outil pour accroître les compétences interculturelles, renforcer la coopération mondiale et découvrir des façons nouvelles et innovantes de penser et de travailler ensemble (OCDE, à paraître).

Conscients de ces atouts, de nombreux pays accordent de plus en plus d'importance à l'enseignement des langues étrangères.

\section{Qu'est-ce que l'Enquête TALIS ?}

Lancée en 2008, l'Enquête internationale sur l'enseignement et l'apprentissage (TALIS) est la première grande étude internationale auprès d'enseignants et de chefs d'établissement qui examine différents aspects ayant une incidence sur l'apprentissage des élèves. Elle donne une voix aux enseignants et aux chefs d'établissement, leur permettant de contribuer à l'analyse et au développement des politiques en éducation dans des domaines clés.

Les enseignants et les chefs d'établissement en poste dans le secteur public et privé dans le premier cycle de l'enseignement secondaire ordinaire constituent la population internationale cible de TALIS 2018. Dans chaque pays, un échantillon représentatif de 4000 enseignants, ainsi que leur chef d'établissement, a été prélevé de manière aléatoire dans 200 établissements, en vue de l'Enquête de 2018. Environ 260000 enseignants ont fourni des réponses en lien avec toutes les composantes de l'enquête, ce qui représente plus de 8 millions d'enseignants de 48 pays et économies participant à l'Enquête.

Le site de l'Enquête TALIS (www.oecd.org/education/talis) fournit de plus amples informations. 
Ce numéro de la série L'enseignement à la loupe se propose d'explorer la spécialisation, la formation et la mobilité des professeurs de langues étrangères. Par " professeurs de langues étrangères ", on entend ici les enseignants du premier cycle du secondaire déclarant enseigner " les langues vivantes étrangères ", indépendamment des autres catégories de matières qu'ils enseignent'.

\section{Les professeurs de langues étrangères constituent une part importante du corps enseignant}

L'enquête TALIS demande aux enseignants d'indiquer la ou les catégories de matières qu'ils enseignent pendant l'année scolaire en cours. Ils ont la possibilité de choisir n'importe quelle combinaison dans une liste de 12 catégories, dont « les langues vivantes étrangères », « la lecture, l'expression écrite et la littérature » dans la langue maternelle des élèves ou la langue d'enseignement, « les mathématiques », « les sciences » et «le grec ancien et/ou le latin ».

Le graphique 1 montre la proportion d'enseignants indiquant enseigner les langues vivantes étrangères dans l'Enquête TALIS 2018, par rapport aux autres matières qu'ils enseignent. En moyenne, dans les pays et économies participant à TALIS, $18 \%$ des enseignants enseignent les langues vivantes étrangères, avec d'importantes différences toutefois entre les systèmes d'éducation. En Autriche, au Danemark, en Islande, en Norvège et en République tchèque, au moins 30 \% des enseignants indiquent enseigner les langues vivantes étrangères (dont certains enseignent aussi d'autres matières). Par contraste, cette proportion est inférieure à 10 \% en Afrique du Sud, en Angleterre (Royaume-Uni), en Australie et aux États-Unis.

En moyenne, dans les pays et économies participant à TALIS, les professeurs de langues étrangères sont plus susceptibles d'être des femmes et légèrement plus jeunes que les autres enseignants (tableaux 1, 2 et $3^{2}$ ). On compte ainsi $22 \%$ d'hommes parmi les professeurs de langues étrangères, contre $33 \%$ parmi les autres enseignants, en moyenne, dans les pays et économies participant à TALIS. La proportion de professeurs de langues étrangères âgés de 40 ans ou plus est de $58 \%$, contre $61 \%$ parmi les autres enseignants.

Seule une minorité des professeurs de langues étrangères sont spécialisés dans cette discipline, c'est-à-dire enseignent uniquement les langues vivantes étrangères (graphique 1). En moyenne, dans les pays et économies participant à TALIS, la proportion d'enseignants spécialisés en langues étrangères est de $7 \%$. Cette proportion est particulièrement élevée dans certains pays européens, comme les Pays-Bas (16 \%) et l'Italie (14 \%).

Graphique 1. Proportion de professeurs de langues étrangères

Pourcentage d'enseignants du premier cycle de l'enseignement du secondaire indiquant enseigner actuellement les catégories de matières suivantes

\footnotetext{
- Professeurs de langues étrangères enseignant aussi d'autres matières

- Professeurs de langues étrangères enseignant aussi la lecture, l'expression écrite et la littérature dans la langue maternelle/la langue d'enseignement

Enseignants spécialisés en langues étrangères
}

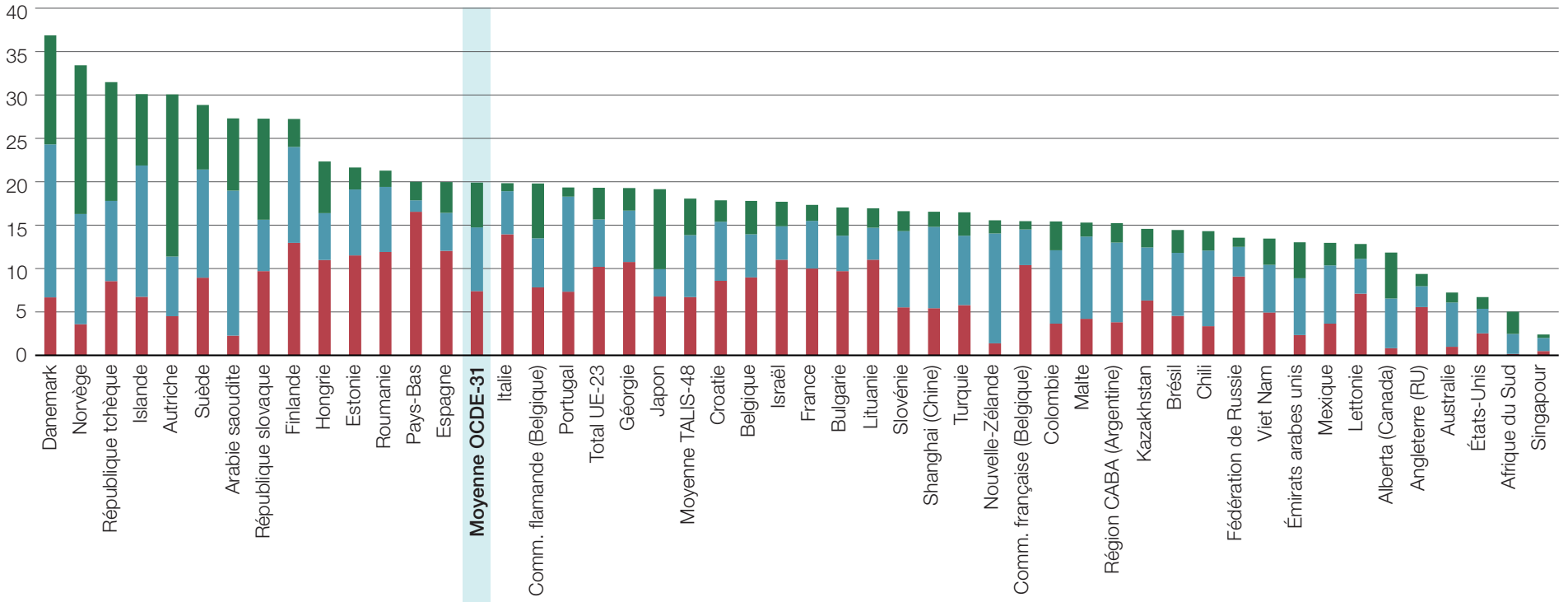

Note : La hauteur totale de la barre représente la proportion totale d'enseignants indiquant enseigner « les langues vivantes étrangères ". Par « enseignants spécialisés en langues étrangères ", on entend ceux indiquant enseigner uniquement les langues vivantes étrangères. Par " professeurs de langues étrangères enseignant aussi d'autres matières ", on entend ceux indiquant enseigner d'autres catégories de matières en plus des langues vivantes étrangères (à l'exception de la catégorie « lecture, expression écrite et littérature ”, représentée séparément dans ce graphique). CABA = Ciudad Autónoma de Buenos Aires (Argentine).

Source : OCDE, Base de données TALIS 2018 ; www.oecd.org/education/talis/TIF 33 Tables Foreign Lanquage Teachers.xlsx, tableau 1. 
La plupart des professeurs de langues étrangères ne sont pas spécialisés et enseignent également d'autres catégories de matières. La catégorie la plus couramment enseignée par les professeurs de langues étrangères est « la lecture, l'expression écrite et la littérature » dans la langue maternelle des élèves ou la langue d'enseignement ( $42 \%$ des professeurs de langues étrangères, en moyenne, dans les pays et économies participant à TALIS) (tableau 4). En moyenne, dans les pays et économies de l'OCDE, les autres matières couramment enseignées par les professeurs de langues étrangères sont « les sciences humaines » (25\%), « les disciplines artistiques » (21\%), « la technologie » (20 \%), ainsi que « les mathématiques » et « les sciences » (toutes deux à $19 \%$ ).

\section{Box 1. Les professeurs de langues étrangères dans le primaire et le deuxième cycle du secondaire}

La proportion d'enseignants spécialisés en langues étrangères est significativement plus faible dans le primaire que dans le premier cycle du secondaire dans 12 des 13 pays et économies dont les données sont disponibles (tableau 1_ 11). Ce constat peut expliquer pourquoi la proportion d'enseignants indiquant enseigner les langues vivantes étrangères est significativement plus élevée dans le primaire dans 9 des 13 pays et économies dont les données sont disponibles (au Danemark, cette proportion est à l'inverse significativement plus faible). Ces différences sont particulièrement notables en France, où $73 \%$ des enseignants du primaire indiquent enseigner les langues vivantes étrangères (contre $17 \%$ dans le premier cycle de l'enseignement du secondaire), mais moins de 1 \% déclarent enseigner uniquement cette catégorie de matières (contre $10 \%$ dans le premier cycle de l'enseignement du secondaire).

Dans 4 des 11 pays et économies dont les données sur le deuxième cycle de l'enseignement du secondaire sont disponibles, on n'observe aucune différence significative avec le premier cycle de l'enseignement du secondaire si on regarde la proportion d'enseignants indiquant enseigner une langue vivante étrangère, ou la proportion d'enseignants spécialisés en langues étrangères (tableau 1_I3). Dans les 7 autres pays et économies, la proportion de professeurs de langues étrangères est plus faible dans le deuxième cycle de l'enseignement du secondaire que dans le premier. Dans 5 de ces 7 pays, la proportion d'enseignants spécialisés en langues étrangères est aussi significativement plus faible dans le deuxième cycle de l'enseignement secondaire que dans le premier cycle de l'enseignement secondaire. À titre d'exemple, en Croatie, $14 \%$ de l'ensemble des enseignants du deuxième cycle de l'enseignement du secondaire déclarent enseigner les langues vivantes étrangères (contre $18 \%$ au niveau 2 de la CITE), et $6 \%$ d'entre eux indiquent enseigner uniquement cette catégorie de matières (contre $9 \%$ au niveau 2 de la CITE).

\section{Les professeurs de langues étrangères reçoivent une formation similaire à celle des autres} enseignants, avec toutefois quelques différences notables de contenu

L'Enquête TALIS demande aux enseignants comment ils ont obtenu leur premier diplôme d'enseignant, et leurs réponses ne mettent au jour aucune différence notable entre les professeurs de langues étrangères et les autres enseignants (tableaux 5 et 6). En moyenne, dans les 32 pays et économies participant à TALIS qui ont administré cette question, une proportion quasi identique de professeurs de langues étrangères (59 \%) et d'autres enseignants (58 \%) indiquent avoir obtenu leur premier diplôme d'enseignant à l'issue d'une formation ou d'études d'enseignant classique(s) simultanée(s), c'est-à-dire un diplôme unique sanctionnant l'étude du contenu de la ou des matières, de la pédagogie et d'autres cours sur l'éducation. Ils sont par ailleurs $24 \%$ parmi les professeurs de langues étrangères comme parmi les autres enseignants à indiquer avoir obtenu leur premier diplôme d'enseignement à l'issue d'une formation ou d'études d'enseignant classique(s) consécutive(s), c'est-à-dire avoir mené à bien deux phases d'enseignement post-secondaire.

L'Enquête TALIS interroge également les enseignants sur les aspects inclus dans leurs études ou leur formation dans le cadre institutionnel, ainsi que sur leur sentiment de préparation à cet égard. Leurs réponses mettent au jour des différences intéressantes (présentées dans le graphique 2) entre les professeurs de langues étrangères et les autres enseignants, même après contrôle de leur âge, de leur sexe, de leur ancienneté et de la proportion d'élèves issus de foyers défavorisés dans leur classe. En moyenne, dans les pays et économies participant à TALIS, les professeurs de langues étrangères sont plus susceptibles (de 9 points de pourcentage) que les autres enseignants de déclarer que «l'enseignement en milieu multiculturel ou plurilingue » figurait au programme de leurs études ou de leur formation dans le cadre institutionnel, et d'indiquer s'estimer « bien » ou "très bien » préparés à ce type d'enseignement. On observe les différences les plus marquées en Amérique latine, où elles sont supérieures à 20 points de pourcentage dans la région de la Ciudad Autónoma de Buenos Aires (ci-après dénommée "région CABA [Argentine] ") et au Chili pour ce qui concerne l'inclusion de l'enseignement en milieu multiculturel ou plurilingue dans les études ou la formation dans le cadre institutionnel, et au Brésil, au Chili, dans la région CABA (Argentine) et au Mexique pour ce qui concerne le sentiment de préparation à l'enseignement en milieu multiculturel ou plurilingue (tableau 7). 
Par contraste, en moyenne, dans les pays et économies de l'OCDE, les professeurs de langues étrangères sont moins susceptibles (de 4 points de pourcentage) que les autres enseignants de déclarer que « l'enseignement de compétences transversales » figurait au programme de leurs études ou de leur formation dans le cadre institutionnel, et moins susceptibles (de 3 points de pourcentage) d'indiquer s'estimer « bien » ou « très bien » préparés à ce type d'enseignement (graphique 2 et tableau 7). Singapour fait à cet égard figure d'exception notable : les professeurs de langues étrangères (qui ne représentent qu'une faible proportion du corps enseignant) y sont plus susceptibles (de 23 points de pourcentage) que les autres enseignants d'indiquer s'estimer préparés à l'enseignement de compétences transversales (comme la créativité ou la résolution de problèmes).

En moyenne, dans les pays et économies de l'OCDE, on n'observe aucune différence significative entre les professeurs de langues étrangères et les autres enseignants pour la plupart des autres aspects de l'enseignement (où quand différence significative il y a, elle n'excède jamais 2 points de pourcentage) (graphique 2).

Graphique 2. Contenu de la formation des enseignants et sentiment de préparation de ces derniers à différents aspects de leur métier

Différence (exprimée en points de pourcentage) entre les professeurs de langues étrangères et les autres enseignants du premier cycle de l'enseignement du secondaire, moyenne TALIS

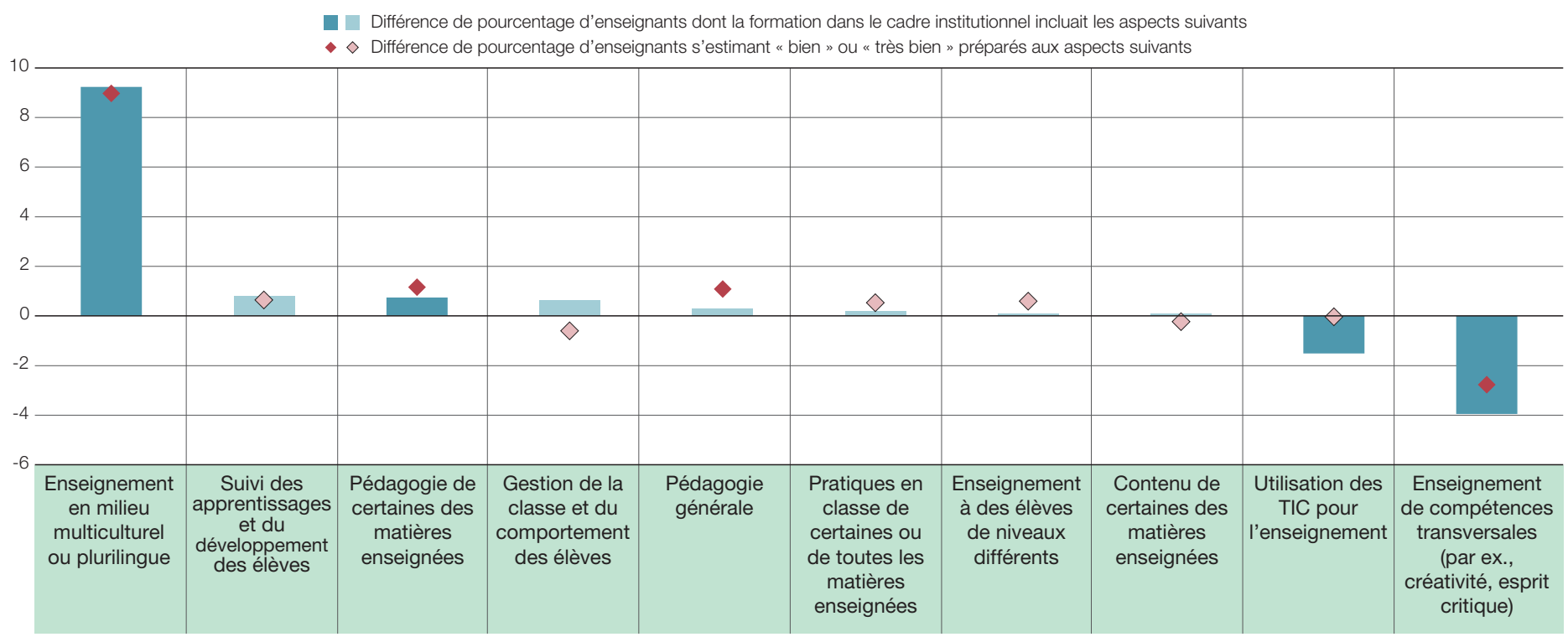

Note : Les barres et les losanges colorés représentent les différences significatives. Les différences (exprimées en points de pourcentage) entre les professeurs de langues étrangères et les autres enseignants sont obtenues par des modèles de probabilité linéaire dans lesquels on opère une régression d'une variable binaire pour l'inclusion d'un certain aspect dans la formation de l'enseignant ou son sentiment de préparation sur une variable binaire relative au fait d'être professeur de langues étrangères. L'âge des enseignants, leur sexe, leur ancienneté dans l'établissement à l'étude, ainsi que le pourcentage indiqué d'élèves issus de foyers défavorisés dans leur classe, sont inclus à titre de variables de contrôle.

Source : OCDE, Base de données TALIS 2018 ; www.oecd.org/education/talis/TIF 33 Tables Foreign Language Teachers.xlsx, tableau 7.

\section{Les professeurs de langues étrangères ont une grande variété d’expériences internationales}

L'Enquête TALIS interroge les enseignants sur leur mobilité internationale, et leurs réponses mettent au jour de profondes différences à cet égard entre les professeurs de langues étrangères et les autres enseignants dans de nombreux pays et économies. Ces questions étaient facultatives dans l'Enquête : 36 pays et économies ont administré celle sur la mobilité globale des enseignants et 34, celle sur les objectifs de cette mobilité.

En moyenne, dans les 36 pays et économies participant à TALIS qui ont administré cette question, $34 \%$ des professeurs de langues étrangères sont allés à l'étranger faire des études pendant leur formation initiale d'enseignant, contre $14 \%$ des autres enseignants (graphique 3). Cette différence est particulièrement marquée dans l'Union européenne, où la proportion de professeurs de langues étrangères ayant fait des études à l'étranger pendant leur formation initiale d'enseignant s'élève à $52 \%$, contre seulement $18 \%$ pour les autres enseignants (estimation basée sur les pays de TALIS membres de l'Union européenne) (tableau 8).

Les professeurs de langues étrangères sont bien plus susceptibles (de 25 points de pourcentage) que les autres enseignants d'indiquer être allés à l'étranger pour apprendre des langues, mais aussi plus susceptibles (de 6 points de pourcentage) d'y être allés pour apprendre à enseigner d'autres matières. En outre, les professeurs de langues étrangères sont plus susceptibles d'avoir bénéficié d'une expérience d'enseignement dans un pays étranger (graphique 3). En moyenne, dans les 34 pays et économies participant à TALIS qui ont administré cette question, $18 \%$ des professeurs de langues étrangères sont allés enseigner à l'étranger, contre $10 \%$ des autres enseignants. La différence est particulièrement marquée dans 
l'Union européenne, où la proportion de professeurs de langues étrangères ayant enseigné à l'étranger est de 26 \%, contre seulement $9 \%$ parmi les autres enseignants. Dans tous les systèmes d'éducation dont les données sont disponibles, à l'exception du Viet Nam, les professeurs de langues étrangères sont significativement plus susceptibles que les autres enseignants d'être allés apprendre des langues à l'étranger. Dans tous ces systèmes d'éducation à l'exception de l'Alberta (Canada), de la Finlande, de l'Islande, de Malte et du Viet Nam, ils sont aussi significativement plus susceptibles que les autres enseignants d'être allés enseigner à l'étranger (tableaux 11 et 15).

Compte tenu de leur plus forte propension à aller à l'étranger pour des raisons professionnelles, il n'est pas surprenant que les professeurs de langues étrangères aient davantage recours aux dispositifs de mobilité existants. En moyenne, dans les 36 pays et économies participant à TALIS qui ont administré cette question, $13 \%$ des professeurs de langues étrangères sont allés à l'étranger dans le cadre d'un programme régional ou national, contre $8 \%$ des autres enseignants. En outre, $19 \%$ des professeurs de langues étrangères sont allés à l'étranger à titre professionnel dans le cadre d'un programme de l'Union européenne (par ex., Erasmus+, Comenius), contre $8 \%$ des autres enseignants. Les enseignants des pays non européens recourent aussi à ce type de programmes. À titre d'exemple, $13 \%$ des professeurs de langues étrangères des Émirats arabes unis et $11 \%$ de ceux du Mexique indiquent être allés enseigner à l'étranger dans le cadre d'un programme de l'Union européenne (graphique 3 et tableau 9).

Pour les professeurs de langues étrangères, l'étranger représente davantage qu'une possibilité de développement professionnel (graphique 3). Ainsi, $24 \%$ des professeurs de langues étrangères sont allés à l'étranger pour accompagner des élèves en voyage (contre $13 \%$ des autres enseignants), en moyenne, dans les 34 pays et économies participant à TALIS qui ont administré cette question. En outre, $22 \%$ des professeurs de langues étrangères sont allés à l'étranger afin d'établir des contacts avec d'autres établissements scolaires (contre $13 \%$ des autres enseignants). Dans l'Union européenne, $43 \%$ des professeurs de langues étrangères sont allés à l'étranger pour accompagner des élèves en voyage et $29 \%$, pour établir des contacts avec d'autres établissements scolaires. À titre de comparaison, $16 \%$ des autres enseignants sont allés à l'étranger pour accompagner des élèves en voyage et $12 \%$ pour établir des contacts avec d'autres établissements scolaires (tableaux 13 et 14).

Graphique 3. Professeurs de langues étrangères et autres enseignants qui sont allés à l'étranger à titre professionnel, selon le motif de ce séjour

Pourcentage d'enseignants du premier cycle de l'enseignement du secondaire qui sont allés à l'étranger à titre professionnel pendant leurs études ou leur formation, ou une fois devenus enseignants, moyenne TALIS basée sur les pays et économies ayant administré ces questions.

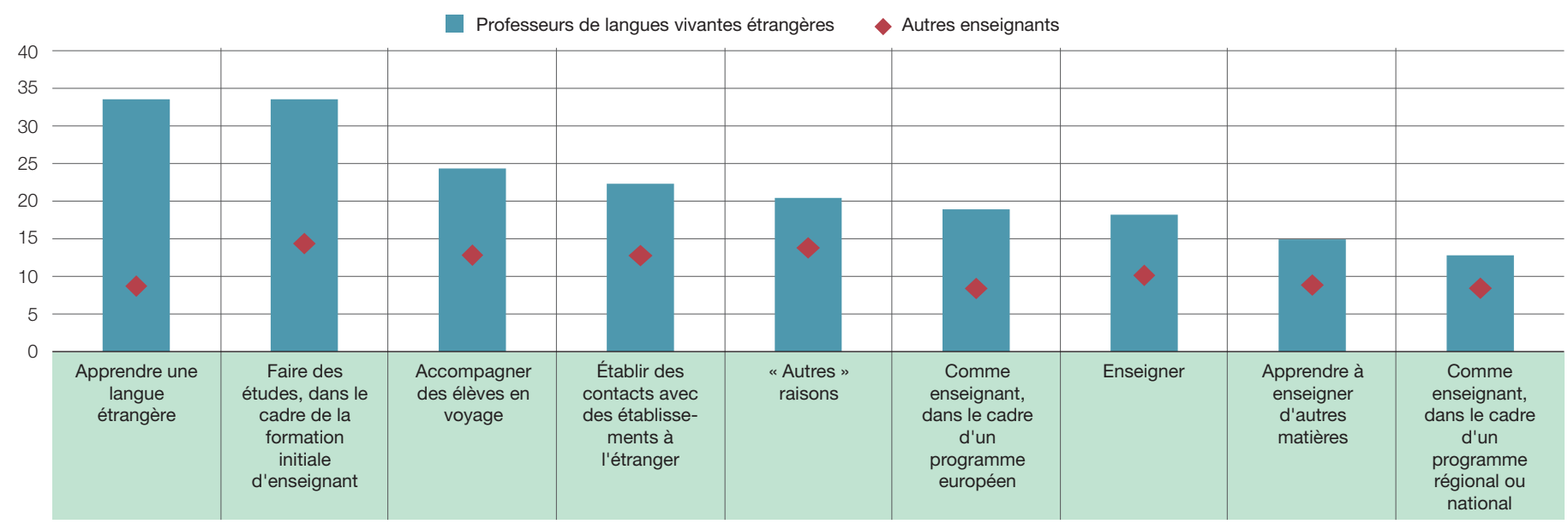

Note : Les différences entre les professeurs de langues étrangères et les autres enseignants sont toutes significatives. Pour les séries « Pendant ma formation initiale d'enseignant ", " Comme enseignant, dans le cadre d'un programme européen » et « Comme enseignant, dans le cadre d'un programme régional ou national ", la moyenne se rapporte aux 36 pays et économies qui ont administré la question sur la mobilité globale ; pour toutes les autres séries, la moyenne se rapporte aux 34 pays qui ont administré la question sur les objectifs de la mobilité.

Source : OCDE, Base de données TALIS 2018 ; www.oecd.org/education/talis/TIF 33 Tables Foreign Language Teachers.xlsx, tableaux 8, 9, 10, 11, 12, 13, 14, 15 et 16.

\section{Quel sera l'impact des perturbations provoquées par la COVID-19 sur les professeurs de langues étrangères?}

La pandémie actuelle de COVID-19 a des répercussions sur les déplacements internationaux et continuera probablement d'en avoir à court et à moyen terme. Les professeurs de langues étrangères verront une partie de leurs rôles fortement affectée et devront, pour poursuivre leur orientation internationale, trouver d'autres stratégies que les voyages. L'utilisation des TIC peut aider à résoudre ce problème, étant donné leur potentiel en termes de communication à longue distance et de connexion mondiale. Les enseignants peuvent se connecter en ligne avec des pairs d'autres pays, notamment en 
participant à des réseaux professionnels. En moyenne, dans les pays et économies participant à TALIS, un pourcentage quasi identique de professeurs de langues étrangères (49 \%) et d'autres enseignants (48 \%) avaient participé, au cours des 12 derniers mois, à un réseau d'enseignants spécifiquement axé sur développement professionnel (tableau 18). En outre, $42 \%$ des professeurs de langues étrangères ont indiqué avoir participé à des cours ou des séminaires en ligne au cours des 12 derniers mois (contre $40 \%$ des autres enseignants) (tableau 17).

En intégrant les TIC dans leur enseignement, les enseignants peuvent permettre aux élèves d'accéder à des contenus d'autres cultures et communautés linguistiques, quelles que soient les restrictions de déplacement en vigueur. Dans les pays et économies participant à TALIS, les professeurs de langues étrangères s'estiment aussi bien préparés que les autres enseignants à l'utilisation des TIC pour leur enseignement (graphique 2). Ils sont toutefois plus susceptibles d'utiliser les TIC en classe. En moyenne, dans les pays et économies ayant participé à TALIS 2018, 62 \% des professeurs de langues étrangères indiquent laisser " souvent » ou " toujours » les élèves utiliser les TIC pour des projets ou des travaux en classe, contre $56 \%$ des autres enseignants (tableau 19). Des différences particulièrement marquées entre professeurs de langues étrangères et autres enseignants s'observent à cet égard au Brésil (21 \%) et en Suède (14 \%).

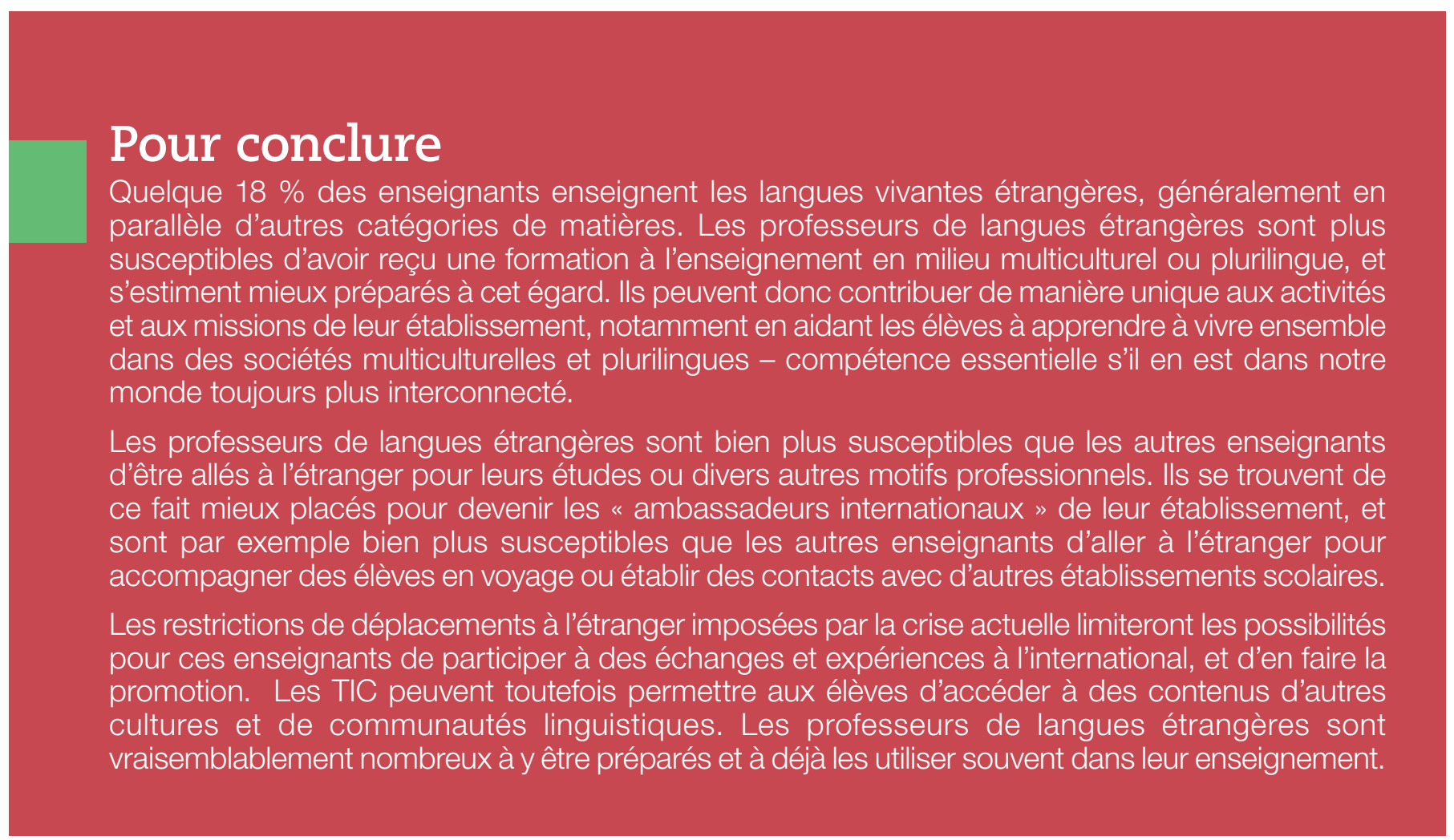

\section{Notes}

1. Dans ce numéro de la série L'enseignement à la loupe, la moyenne TALIS est une estimation de la moyenne arithmétique des données se rapportant aux enseignants du premier cycle de l'enseignement secondaire dans les 48 pays et économies de l'OCDE participant à TALIS. Cependant, certains items n'ont été administrés que par quelques pays et économies. Ces exceptions sont dûment signalées dans les sections concernées. Dans la dernière section de ce numéro, un total « Union européenne " est estimé sur la base des données des enseignants du premier cycle de l'enseignement secondaire des pays membres ayant administré l'item concerné.

2. Les tableaux mentionnés dans ce numéro de la série L'enseignement à la loupe sont consultables sur : www.oecd.org/education/talis/TIF_33_Tables_Foreign_Language_Teachers.xlsx (disponible uniquement en anglais). 


\section{Personnes à contacter}

Gabriele Marconi (Gabriele.Marconi@oecd.org) et Catalina Covacevich (Catalina.Covacevich@oecd.org)

\section{Pour en savoir plus}

OCDE (à paraître), PISA 2024 Foreign Language Assessment Background Questionnaires Framework, Paris, OCDE.

Les tableaux mentionnés dans ce numéro de la série L'enseignement à la loupe sont consultables sur :

www.oecd.org/education/talis/TIF 33 Tables Foreign Language Teachers.xlsx (disponible uniquement en anglais).

La publication de ce document relève de la responsabilité du Secrétaire général de l'OCDE. Les opinions et les interprétations exprimées ne reflètent pas nécessairement les vues des pays membres de l'OCDE.

Ce document, ainsi que les données et cartes qu'il peut comprendre, sont sans préjudice du statut de tout territoire, de la souveraineté s'exerçant sur ce dernier, du tracé des frontières et limites internationales, et du nom de tout territoire, ville ou région.

Les données statistiques concernant Israël sont fournies par et sous la responsabilité des autorités israéliennes compétentes. L'utilisation de ces données par l'OCDE est sans préjudice du statut des hauteurs du Golan, de Jérusalem-Est et des colonies de peuplement israéliennes en Cisjordanie aux termes du droit international.

L'utilisation de ce travail, sous forme numérique ou papier, est régie par les conditions d'utilisation consultables sur : http://www.oecd.org/fr/conditionsdutilisation/

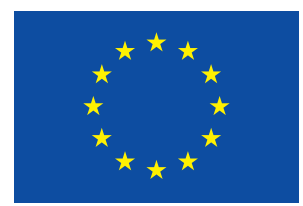

Co-financé par l’Union européenne
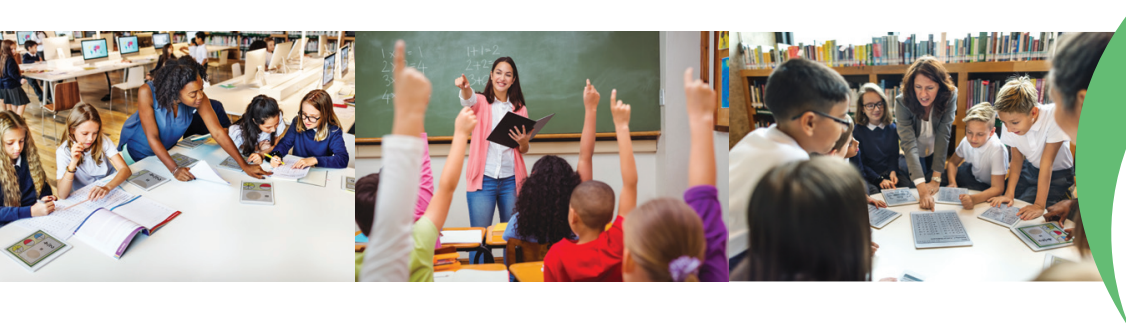